\title{
Detection and Continuation of a Border Collision Bifurcation in
} a Forest Fire Model

Fabio Dercole (dercole@elet.polimi.it)

Stefano Maggi (maggi@elet.polimi.it)

\section{Approved by}

Ulf Dieckmann

Program Leader, Evolution \& Ecology

December 2005 Institute, its National Member Organizations, or other organizations supporting the work. 


\section{Contents}

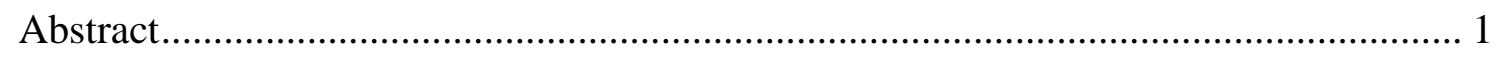

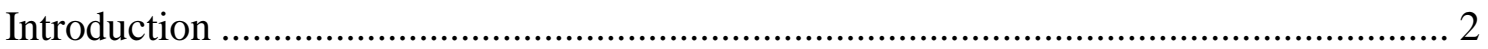

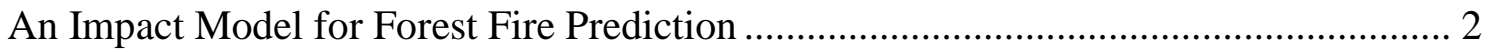

Characteristic Bifurcations of the Impact Model .......................................................... 5

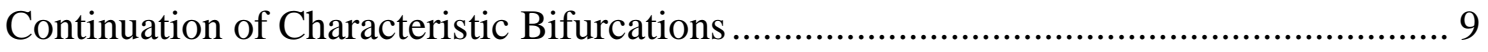

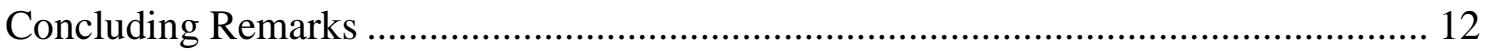

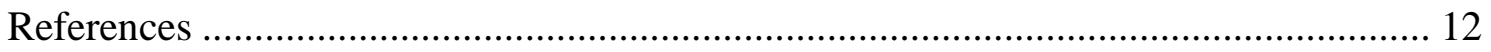




\title{
Detection and continuation of a border collision bifurcation in a forest fire model
}

\author{
Fabio Dercole *, Stefano Maggi \\ Department of Electronics and Information, Politecnico di Milano, \\ Via Ponzio 34/5, IT-20133 Milano, Italy
}

\begin{abstract}
The behavior of the simplest forest fire model is studied in this paper through bifurcation analysis. The model is a second-order continuous-time impact model where vegetational growth is described as a continuous and slow dynamic process, while fires are modeled as instantaneous and disruptive events. The transition from Mediterranean forests (characterized by wild chaotic fire regimes) to savannas and boreal forests (where fires are almost periodic) is recognized to be a catastrophic transition known as border collision bifurcation in the context of discrete-tine systems. In the present case such a bifurcation can be easily detected numerically and then continued by solving a standard boundary-value problem. The result of the analysis complements previous simulation studies and are consistent with biological intuition.

(C) 2004 Elsevier Inc. All rights reserved.
\end{abstract}

Keywords: Border collision bifurcation; Chaos; Continuation; Forest fire model; Impact model

\footnotetext{
${ }^{*}$ Corresponding author.

E-mail address: dercole@elet.polimi.it (F. Dercole).
} 


\section{Introduction}

Border collision bifurcations have been defined for continuous piecewise smooth maps depending on parameters [1-6]. In the simplest case of onedimensional maps, border collision bifurcations occur, as a parameter is varied, when a fixed or periodic point of the map collides with the set of points (called border) where the map is not differentiable.

Bifurcations similar to border collision bifurcations also arise in discontinuous piecewise smooth vector fields, called Filippov systems [7,8], in switched systems (which include continuous piecewise smooth maps, see e.g. [9]), in evolutionary systems [10], and, as shown in this paper, in impact systems (see e.g. [11, Chapter 7]), described by smooth vector fields whose orbits undergo abrupt jumps in state space.

Obviously, the consequences of a border collision bifurcation on the system behavior strongly depend on the considered class of systems. However, the detection and continuation of the bifurcation in parameter space pose the same problems. The aim of this paper is to show, through the analysis of a secondorder continuous-time impact model, how the continuation of border collision bifurcations can be performed by solving standard boundary-value problems.

The model considered in the following describes the dynamics of forest fires and its bifurcation analysis complements the simulation study carried out in [12]. As we will see, the key for understanding the transition from chaos to cycles is, indeed, a border collision bifurcation.

\section{An impact model for forest fire prediction}

While it is true that natural forest fires originate from random events (mostly lightning) and can easily develop under specific meteorological conditions [13], it is also true that relevant fires can occur only if there is enough fuel, i.e. only if at least one of the various vegetational layers of the forest is sufficiently rich. This suggests the idea that long-term predictions of forest fires can be performed with deterministic models describing the growth processes, while more precise short-term predictions can only be performed through stochastic models for weather forecast.

The best known deterministic model for long-term fire prediction is a fourth-order continuous-time model characterized by standard bifurcations [14]. Very recently, a simpler second-order impact model for the long-term prediction of forest fires has been proposed [12]. In this model, the vegetational growth is described by two standard ordinary differential equations (one for the surface layer (bush) and one for the upper layer (trees)), while fire episodes are modeled as instantaneous events. The fire occurs when the biomasses of the two layers reach pre-specified values. The consequence of a fire is therefore an 
instantaneous reduction of the biomasses which is described, quite empirically, by a simple map in state space. Models with discontinuities of this kind have been used almost exclusively to analyze mechanical systems with impacts (e.g. the motion of the ping-pong ball) and are called, for this reason, impact models.

A continuous-time model is, in general, described by a set of $n$ ordinary differential equations

$$
\dot{x}=f(x),
$$

where $x$ is the $n$-dimensional state vector $\left(x_{1}, x_{2}, \ldots, x_{n}\right)$. In standard models Eq. (1) holds at any point in state space. By contrast, in impact models Eq. (1) holds in all points except on a $(n-1)$-dimensional manifold $\mathscr{X}^{-}$, where the impact occurs. When the state $x$ reaches the manifold $\mathscr{X}^{-}$at point $x^{-}$, an instantaneous transition described by a map

$$
x^{+}=\varphi\left(x^{-}\right), \quad x^{-} \in \mathscr{X}^{-},
$$

occurs. The set

$$
\mathscr{X}^{+}=\varphi\left(\mathscr{X}^{-}\right)
$$

is the set of the states of the system immediately after the impact. For this reason, the sets $\mathscr{X}^{-}$and $\mathscr{X}^{+}$are called pre- and post-impact manifolds. In our case they simply represent the pre- and post-fire conditions of the forest.

In the model considered in this paper, the forest has two layers: a lower vegetational layer that, depending on the forest, is composed of bryophytes, herbs, shrubs, or any mix of these plants, and an upper vegetational layer, in general composed of trees of various species. The corresponding biomasses are denoted by $B$ (bush) and $T$ (tree). The equations of growth (1) characterizing the model are

$$
\begin{aligned}
& \dot{B}=r_{B} B\left(1-\frac{B}{K_{B}}\right)-\alpha B T, \\
& \dot{T}=r_{T} T\left(1-\frac{T}{K_{T}}\right) .
\end{aligned}
$$

This means that, in the absence of fire, trees grow logistically toward the carrying capacity $K_{T}$, while plants of the lower layer do not tend toward their carrying capacity $K_{B}$ because tree canopy reduces light availability. A detailed discussion of the validity of Eq. (3) can be found in [14], where realistic values for the five vegetational parameters $\left(r_{B}, r_{T}, K_{B}, K_{T}, \alpha\right)$ are also suggested.

The pre- and, twice post-impact manifolds $\mathscr{X}^{-}$and $\mathscr{X}^{+}$(i.e. the pre-and postfire conditions) characterizing map (2) are described in Fig. 1(a).

Let us first focus on the pre-fire conditions by noticing that the function $T(B)$ identifying the manifold $\mathscr{X}^{-}$is non-increasing and that the set below the manifold $\mathscr{X}^{-}$is convex. The first property is needed because less trees 

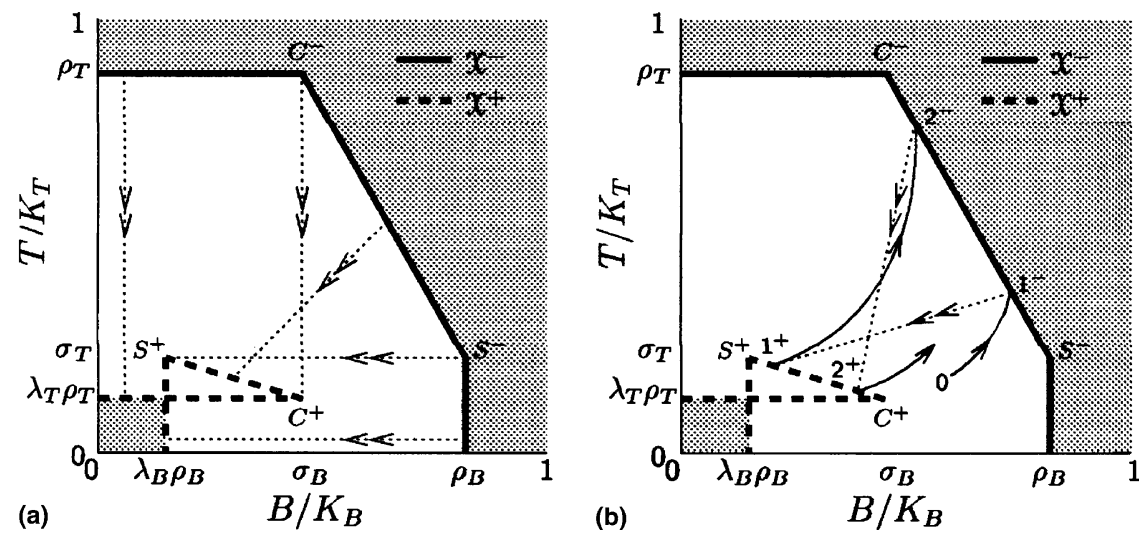

Fig. 1. Model behavior. (a) The pre- and post-fire manifolds $\mathscr{X}^{-}$and $\mathscr{X}^{+}$; the dotted lines with double arrows are the instantaneous transitions from $\mathscr{X}^{-}$to $\mathscr{X}^{+}$due to a fire (see Eq. (2)); horizontal [vertical] lines correspond to surface [crown] fires in which trees [bush] are not involved; oblique lines starting from the segment $C^{-} S^{-}$of $\mathscr{X}^{-}$correspond to mixed fires. (b) State portrait of the model; continuous lines with a single arrow represent the growing phase of the forest and are described by Eq. (3).

axe necessary for fire ignition if more biomass is available in the lower layer of the forest. The second property simply says that if $x^{\prime}=\left(B^{\prime}, T^{\prime}\right)$ and $x^{\prime \prime}=\left(B^{\prime \prime}, T^{\prime}\right)$ are two states of the forest at which fire ignition is not possible (i.e. two points below the manifold $\mathscr{X}^{-}$) no mix of these two states (i.e. no points of the segment connecting $x^{\prime}$ with $x^{\prime \prime}$ ) can give rise to fire ignition. A formal support of these two properties is given in [12], where it is also shown that $\mathscr{X}^{-}$should be a smooth manifold, while in Fig. 1 it is a piecewise linear manifold. The reason for this choice of $\mathscr{X}^{-}$, geometrically identified by 4 parameters $\left(\rho_{B}, \rho_{T}, \sigma_{B}, \sigma_{T}\right)$, allows one to identify surface fires (vertical segment of $\left.\mathscr{X}^{-}\right)$, crown fires (horizontal segment of $\mathscr{X}^{-}$) and mixed fires (central segment of $\mathscr{X}^{-}$). By definition, surface fires do not involve trees, so that the post-fire conditions are on the vertical segment characterized by $B^{+}=\lambda_{B} \rho_{B} K_{B}=\lambda_{B} B^{-}$. In other words, $\lambda_{B}$ is the proportion of surface biomass that survives to fire. Similarly, crown fires are characterized by a vertical instantaneous transition from $T^{-}=\rho_{T} K_{T}$ to $T^{+}=\lambda_{T} T^{-}$. The surface fire with the highest tree density is represented (see Fig. 1(a)) by the transition $S^{-} \rightarrow S^{+}$, while the crown fire with the highest bush density is represented by the transition $C^{-} \rightarrow C^{+}$. The assumption that mixed fires initiate on the segment $C^{-} S^{-}$implies, by continuity, that postfire conditions are on a curve connecting points $C^{+}$and $S^{+}$. Of course, mixed fires initiating close to point $C^{-}\left[S^{-}\right]$should end up close to point $C^{+}\left[S^{+}\right]$. For simplicity, it is assumed that mixed-fires initiating on the segment $C^{-} S^{-}$at relative distances $(1-\eta)$ and $\eta$ from the two extreme points $C^{-}$and $S^{-}$terminate on the segment $\mathrm{C}^{+} \mathrm{S}^{+}$and preserve the relative distances from the two corre- 
sponding extreme points $C^{+}$and $S^{+}$. In formulas, the extreme points of the prefire segment $C^{-} S^{-}$are

$$
C^{-}=\left(\sigma_{B} K_{B}, \rho_{T} K_{T}\right), \quad S^{-}=\left(\rho_{B} K_{B}, \sigma_{T} K_{T}\right),
$$

and each mixed fire starts from a point $\left(B^{-}, T^{-}\right)$belonging to the segment $C^{-} S^{-}$, i.e.

$$
B^{-}=\eta \sigma_{B} K_{B}+(1-\eta) \rho_{B} K_{B}, \quad T^{-}=\eta \rho_{T} K_{T}+(1-\eta) \sigma_{T} K_{T},
$$

where $0 \leqslant \eta \leqslant 1\left(\eta=0\right.$ and $\eta=1$ correspond to points $S^{-}$and $C^{-}$, respectively). The extreme points of the post-fire segment $C^{+} S^{+}$are

$$
C^{+}=\left(\sigma_{B} K_{B}, \lambda_{T} \rho_{T} K_{T}\right), \quad S^{+}=\left(\lambda_{B} \rho_{B} K_{B}, \sigma_{T} K_{T}\right),
$$

and the post-fire conditions are

$$
B^{+}=\eta \sigma_{B} K_{B}+(1-\eta) \lambda_{B} \rho_{B} K_{B}, \quad T^{+}=\eta \lambda_{T} \rho_{T} K_{T}+(1-\eta) \sigma_{T} K_{T} .
$$

Thus, the map $x^{+}=\varphi\left(x^{-}\right)$for mixed fires is nothing but the transformation of point $\left(B^{-}, T^{-}\right)$into point $\left(B^{+}, T^{+}\right)$.

The evolution of the forest and, in particular, the sequence of the fires can be easily obtained from the model, as shown in Fig. 1(b). Starting from a given initial condition, say point 0 in Fig. 1(b), one integrates the differential equations (3) until the solution hits the pre-fire manifold $\mathscr{X}^{-}$at point $\left(B^{-}, T^{-}\right)$ (see point $1^{-}$in Fig. 1(b)). From anyone of the two equations (5) one can derive the value of $\eta$ associated with point $1^{-}$and then use Eq. (7) for computing point $1^{+}$. Then, the procedure is iterated and a series of fires $2^{-} \rightarrow 2^{+}$, $3^{-} \rightarrow 3^{+} \ldots$ is obtained.

As pointed out in Fig. 1(b), the trajectory of the system is the concatenation of slow transitions (continuous lines) corresponding to growing phases, and fast (actually instantaneous) transitions (dotted lines) corresponding to fires. While slow transitions cannot intersect one each other, fast transitions can intersect with slow and fast transitions, as shown in Fig. 1(b). This is why the model can be chaotic even if it is only a second-order model.

The analysis carried out in [12] has shown, through simulation, that the second-order impact model can mimic very well the qualitative features of the periodic fire regimes of savannas and boreal forests, as well as the chaotic fire regimes of Mediterranean forests. However, much more can be understood through the bifurcation analysis of the model. As we will see in the next sections, questions like "why a chaotic fire regime can become periodic by varying a parameter?" can be easily answered provided a special bifurcation can be detected and continued.

\section{Characteristic bifurcations of the impact model}

We now study the bifurcations of the model described in the previous section. 
If we consider the extreme case of not inflammable forests, i.e. the case in which the pre-fire manifold $\mathscr{X}^{-}$(see Fig. 1) is at infinity, the model is simply described by Eq. (3). Such a model has four equilibria

(i) $B=0, T=0$, absence of vegetation;

(ii) $B=K_{B}, T=0$, bush without trees;

(iii) $B=0, T=K_{T}$, trees without bush;

(iv) $B=\left(1-\frac{\alpha K_{T}}{r_{B}}\right) K_{B}, T=K_{T}$, bush and trees.

The analysis of the Jacobian matrix at these equilibria allows one to conclude that

(i) is always an unstable node;

(ii) is always a saddle;

(iii) is a saddle if $\alpha<\left(r_{B} / K_{T}\right)$ and a stable node otherwise;

(iv) is positive if and only if $\alpha<\left(r_{B} / K_{T}\right)$.

Moreover, (iv) is a stable node when it is positive i.e. when $\alpha<\left(r_{B} / K_{T}\right)$. The results of this preliminary analysis are summarized in Fig. 2 where the two possible state portraits are shown: for $\alpha<\left(r_{B} / K_{T}\right)$, i.e. for low shading effect, bush and trees coexist, while for $\alpha>\left(r_{B} / K_{T}\right)$, i.e. when the shading effect is high, only trees can persist. The connections between the equilibria prove that limit cycles cannot exist. Thus, if bush and trees are not inflammable, there is only one possible bifurcation, namely a transcritical bifurcation of equilibria, which indeed separates the two cases depicted in Fig. 2. At this bifurcation $\alpha=\left(r_{B} / K_{B}\right)$, and the equilibria (iii) and (iv) collide and exchange their stability.

If vegetation is inflammable, the pre-fire manifold $\mathscr{X}^{-}$becomes important and is, indeed, involved in all bifurcations of the impact model. The most obvious bifurcation occurs when the positive equilibrium (iv) touches the pre-fire manifold $\mathscr{X}^{-}$. If, increasing a parameter $p$, the equilibrium (iv) tends to $\mathscr{X}^{-}$from below and crosses $\mathscr{X}^{-}$at $p^{-}$, then for $p>p^{-}$the forest will undergo recurrent fires. This is the case in which we are interested, since our aim is the description of the fire regimes of savannas, boreal forests and Mediterranean forests.

When $p>p^{-}$, namely when the equilibrium (iv) is above the manifold $\mathscr{X}^{-}$, the attractor of the impact model can be a cycle. The simplest case is that of a period- 1 cycle touching the pre-fire manifold $\mathscr{X}^{-}$at a single fire point, as shown in the first row of Fig. 3. If the fire point of the cycle is on the lower [upper] part of the central segment of $\mathscr{X}^{-}$, as in Fig. 3(a) and (b), the fire occurs at high bush [tree] density as in savannas [boreal forests]. But periodic fire regimes can also be period- $k$ cycles with $k \geqslant 2$.

For $p>p^{-}$the attractor can also be a strange attractor characterized by an infinity of fire points on $\mathscr{X}^{-}$, as shown in the second row of Fig. 3. The tran- 
(a)

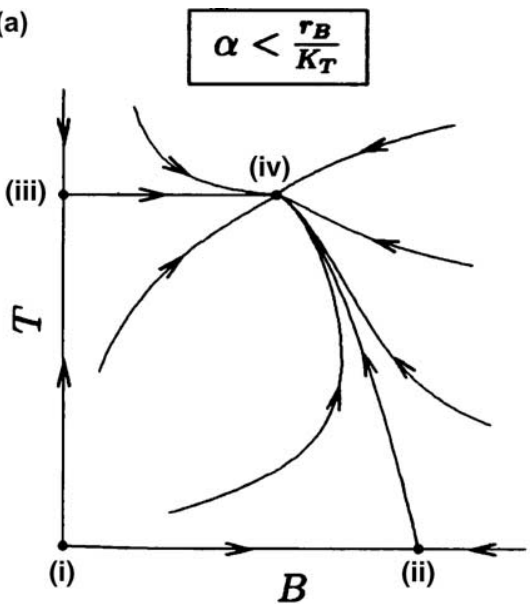

(b)

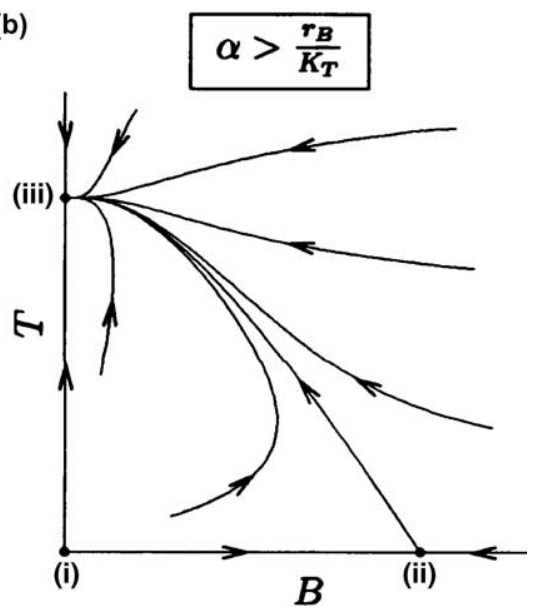

Fig. 2. State portraits in the case of not inflammable forests. (a) Low shading effect; (b) high shading effect. Parameter values: (a) $r_{B}=0.3, r_{T}=0.2, K_{B}=1, K_{T}=1, \alpha=0.4$; (b) as in (a) except $\alpha=1.1$.

sition from a cycle to a strange attractor and the reverse one must a priori be a bifurcation or a sequence of bifurcations. In standard systems a sequence of flip bifurcations (Feigenbaum cascade) is the most common route to chaos $[15,16]$, but this is not so in the present case. This is easy to confirm by producing (through simulation) simple bifurcation diagrams of the kind shown in Fig. 4(a), where pre-fire tree densities $T^{-}$of the fires characterizing a cycle or a strange attractor are plotted as a function of a single parameter $p\left(p=\lambda_{B}\right.$ in Fig. 4(a)). The figure shows that the strange attractor is initially a single-band strange attractor (as in Fig. 3(c)) in which fires alternate in an apparently random fashion with a continuum of bush and tree mix. Increasing the parameter, the attractor becomes a two-bands attractor (as in Fig. 3(d)) in which fires with high tree density alternate randomly with fires with low tree density. Then, the two-bands shrink until for $p=p^{* *}$ they become two points, i.e. the strange attractor becomes more and more similar to a period-2 cycle and finally degenerates exactly into a period- 2 cycle for $p=p^{* *}$. However, for $p>p^{* *}$ the attractor is unique and is a period- 1 cycle. This means that the transition from chaos to cycles obtained by increasing $p$ through $p^{* *}$ is catastrophic.

As for the period-1 cycle, Fig. 4(a) shows that it remains stable not only for $p>p^{* *}$ but also in the range $\left(p^{*}, p^{* *}\right)$ with $p^{*}$ smaller than $p^{* *}$. At $p^{*}$ there is a subcritical flip bifurcation, so that a drop of $p$ below $p^{*}$ triggers a catastrophic transition from a period- 1 cycle to a strange attractor. Actually, the subcritical flip bifurcation at $p^{*}$ is essential for explaining the bifurcation of the strange attractor at $p^{* *}$. In fact, it is the unstable period- 2 cycle created by the flip 

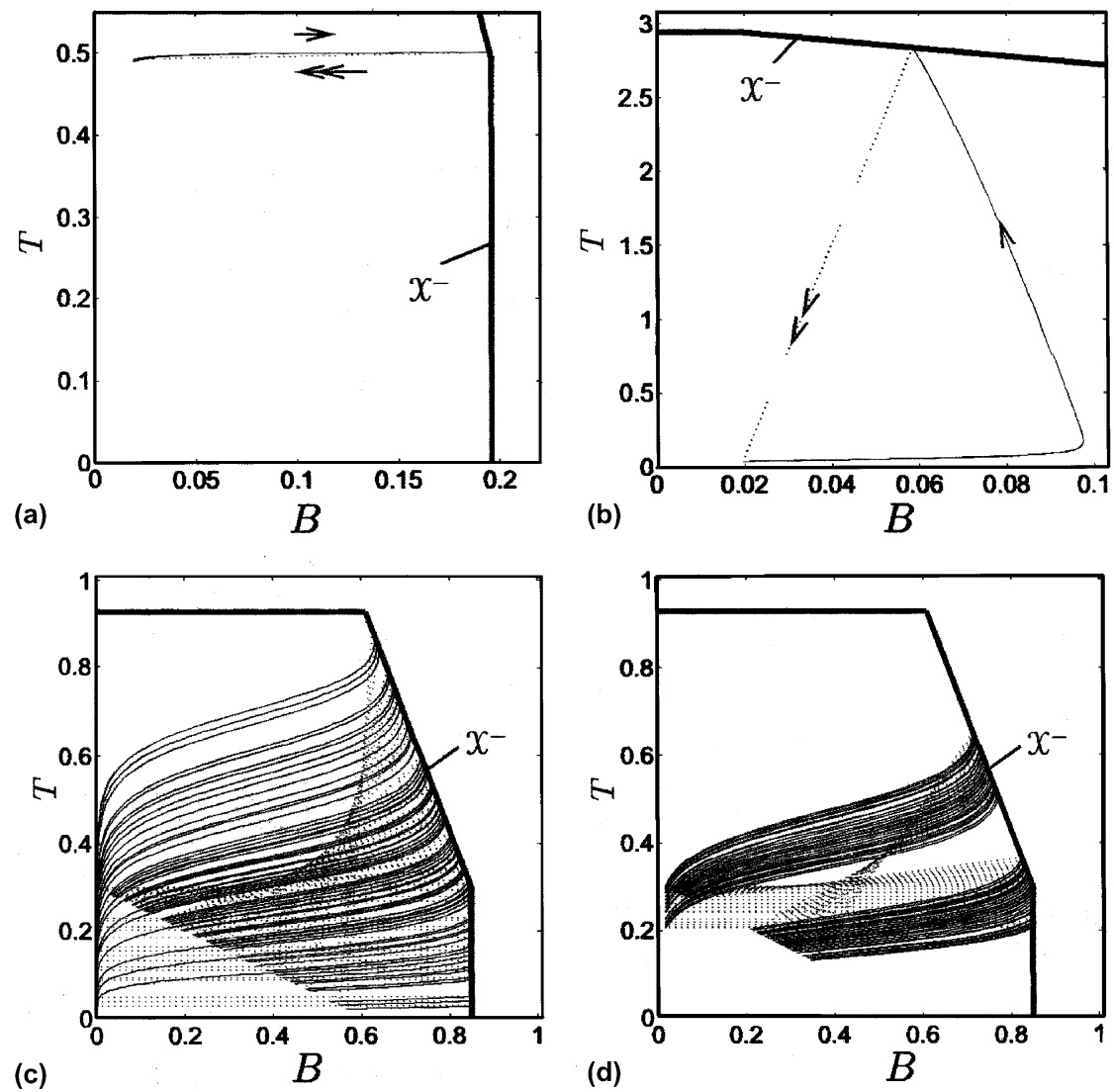

Fig. 3. Attractors of the forest fire model for different parameter settings (see [12]). (a) Period-1 cycle mimicking periodic fire regimes in savannas. (b) Period-1 cycle mimicking periodic fire regimes in boreal forests. (c) Single-band strange attractor mimicking chaotic fire regimes in Mediterranean forests. (d) Two-bands strange attractor mimicking chaotic fire regimes in Mediterranean forests.

bifurcation at $p^{*}$ that for increasing values of $p$ approaches the strange attractor and finally collides with it at $p^{* *}$. Notice that the dashed line tracing the pre-fire tree densities $T$ of the unstable period-2 cycle (see Fig. 4(a)) cannot be obtained by backward simulation, since the model is not reversible. Such a curve has been obtained by numerical continuation [17,18], as explained in the next section.

As a last remark, critically important for the next section, we like to point out that the bifurcation $p^{* *}$ is characterized by the following very peculiar property clearly indicated in Fig. 4(b): one of the two fire points of the period-2 cycle at $p^{* *}$ is one of the two points $C^{-}$and $S^{-}$(see Fig. 1), where the pre-fire manifold $\mathscr{X}^{-}$is not differentiable. Since the one-dimensional map 

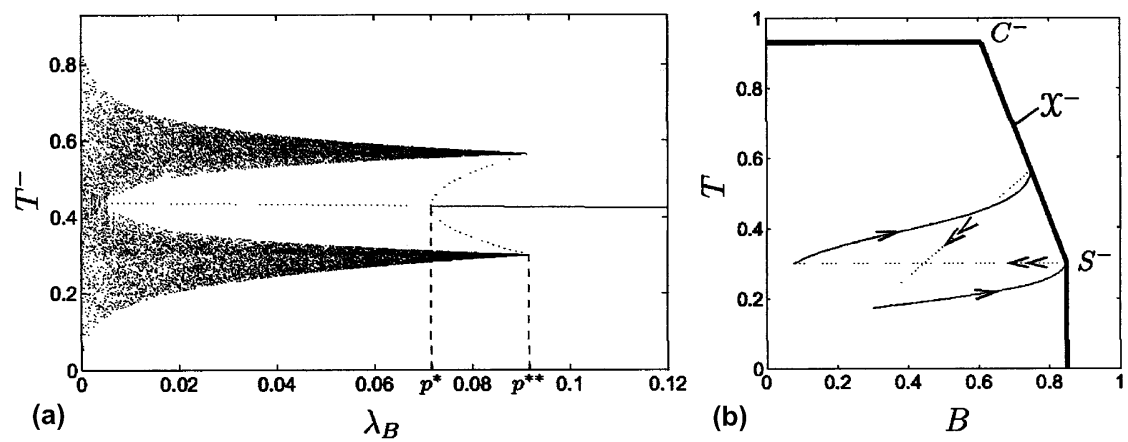

Fig. 4. (a) Bifurcation diagram of the impact model with respect to $\lambda_{B}$ pointing out a subcritical flip bifurcation at $p^{*}$ and a border collision bifurcation and $p^{* *}$. (b) Period-2 cycle passing through point $S^{-}$for $\lambda_{B}=p^{* *}$. Parameter values are $r_{B}=0.375, r_{T}=0.0625, K_{B}=1, K_{T}=1, \alpha=0.43, \rho_{B}=$ $0.85, \rho_{T}=0.93, \sigma_{B}=0.61, \sigma_{T}=0.3, p^{*}=0.0716 \ldots, p^{* *}=0.0916 \ldots, \lambda_{T}=10^{-5}$.

defined on the pre-fire manifold $\mathscr{X}^{-}$by our impact model is not differentiable at points $C^{-}$and $S^{-}$, one can conclude that the transition from the strange attractor to the period- 1 cycle occurring at $p^{* *}$ is, indeed, a border collision bifurcation in the sense of Feigin, Nusse and Yorke.

\section{Continuation of characteristic bifurcations}

This section shows how the flip and border collision bifurcations which occur (see Fig. 4(a)) at $p^{*}$ and $p^{* *}$, respectively, can be continued in the plane of two parameters, say $\left(\lambda_{B}, \lambda_{T}\right)$.

As shown in Fig. 4(b), the border collision bifurcation is characterized by a critical period- 2 cycle having one of the two fire points at $S^{-}$. With reference to Eqs. (1), (2), (4) and (6), such a cycle is a solution of the following boundaryvalue problem in the $[0,1]$ time interval:

$$
\begin{aligned}
& \dot{u}-t_{1} f(u)=0, \\
& \dot{v}-t_{2} f(v)=0, \\
& u_{2}(0)+\frac{S_{2}^{+}-C_{2}^{+}}{C_{1}^{+}-S_{1}^{+}}\left(u_{1}(0)-S_{1}^{+}\right)-S_{2}^{+}=0, \\
& v_{2}(0)+\frac{S_{2}^{+}-C_{2}^{+}}{C_{1}^{+}-S_{1}^{+}}\left(v_{1}(0)-S_{1}^{+}\right)-S_{2}^{+}=0, \\
& u_{2}(1)+\frac{C_{2}^{-}-S_{2}^{-}}{S_{1}^{-}-C_{1}^{-}}\left(u_{1}(1)-C_{1}^{-}\right)-C_{2}^{-}=0,
\end{aligned}
$$




$$
\begin{aligned}
& v_{2}(1)+\frac{C_{2}^{-}-S_{2}^{-}}{S_{1}^{-}-C_{1}^{-}}\left(v_{1}(1)-C_{1}^{-}\right)-C_{2}^{-}=0, \\
& \varphi_{1}(u(1))-v_{1}(0)=0, \\
& \varphi_{1}(v(1))-u_{1}(0)=0, \\
& u_{1}(1)-S_{1}^{-}=0 .
\end{aligned}
$$

Eqs. (8a) and (8b), which are time-scaled versions of Eq. (3), describe the two growing phases, of duration $t_{1}$ and $t_{2}$, respectively, while $u$ and $v$ are twodimensional vectors, whose first and second components represent bush and tree densities. Eqs. (8c)-(8f) say that the two growing phases start and terminate on the straight lines spanned by the central segments $C^{+} S^{+}$and $C^{-} S^{-}$ of $\mathscr{X}^{+}$and $\mathscr{X}^{-}$, respectively. Periodicity is guaranteed by Eqs. $(8 \mathrm{~g})$ and $(8 \mathrm{~h})$, which require that pre-fire conditions $u(1)[v(1)]$ of one phase are mapped by Eq. (2) into post-fire conditions corresponding to the start point $v(0)[u(0)]$ of the other phase. Notice that Eqs. (8g) and (8h) impose this requirement only on the bush variable (first component $\varphi$ ), since start and end points of both growing phases are already constrained by Eqs. (8c)-(8f). Finally, Eq. (8i) imposes that the first growing phase terminates at the critical point $S^{-}$. The whole periodic solution is then given by:

$$
(B(t), T(t))=\left\{\begin{array}{l}
u\left(\frac{t}{t_{1}}\right), \quad t \in\left[0, t_{1}\right], \\
v\left(\frac{t-t_{1}}{t_{2}}\right), \quad t \in\left[t_{1}, t_{1}+t_{2}\right]
\end{array}\right.
$$

and corresponds to the border collision bifurcation as long as $v(1)$ lies on the central segment of $\mathscr{X}^{-}$, i.e. as long as $0 \leqslant \eta \leqslant 1$ in Eq. (5).

The boundary-value problem (8) is composed of four ordinary differential equations and 7 scalar boundary conditions. Thus, the continuation of a one-dimensional solution family of Eq. (8) generically requires $4+7+1=12$ scalar continuation variables, i.e. $\left(u(0), v(0), u(1), v(1), t_{1}, t_{2}\right)$ plus two model parameters, e.g. $\left(\lambda_{B}, \lambda_{T}\right)$, which trace the border collision bifurcation curve. Starting from the initial solution shown in Fig. 4(b) the corresponding bifurcation curve has been numerically produced by standard continuation techniques $[17,18]$ using the software package AUTO97 [19] and is shown in Fig. 5. It separates region 1 of the $\left(\lambda_{B}, \lambda_{T}\right)$ plane, where the fire regime is a stable period-1 cycle, from region 2, where a stable chaotic regime is also possible.

As already pointed out in Section 2, the basins of attraction of the period-1 cycle and of the strange attractor are separated by an unstable period- 2 cycle involving mixed fires, which is also present in region 2 of Fig. 5. Such a cycle is a solution of the boundary-value problem (8a)-(8h), composed of 4 ordinary differential equations and 6 scalar boundary conditions, and can therefore be 


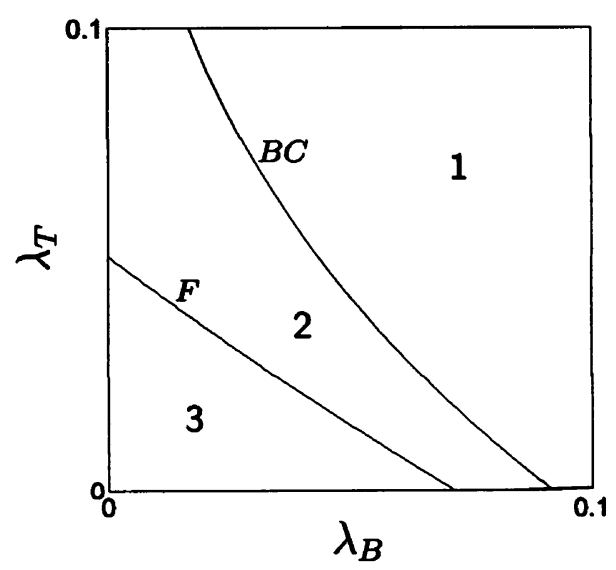

Fig. 5. Bifurcation diagram of the impact model in the $\left(\lambda_{B}, \lambda_{T}\right)$ plane. Other parameter values are as in Fig. 4. Fire regimes are periodic in region 1 and chaotic in region 3, while in region 2 there are two alternative attractors, namely a period- 1 cycle and a strange attractor. Bifurcation curves: BC, border collision; F, subcritical flip bifurcation.

continued with respect to a single model parameter (provided $0 \leqslant \eta \leqslant 1$ for both growing phases along the continuation, see Eq. (5)). Starting again from the initial solution of Fig. 4(b), the unstable period-2 cycle has been continued for decreasing values of $\lambda_{B}$ (actually producing the dashed line tracing pre-fire tree densities $T^{-}$in Fig. 4(b)), until a so-called branch point is detected at $p^{*}$. At a branch point there are two solution families of Eqs. (8a)-(8h) intersecting each other, i.e. the period- 2 cycle collides with a period- 1 cycle traced twice. The branch point thus detects the subcritical flip bifurcation of the period-1 cycle.

The two parameter continuation of the flip bifurcation has been performed by continuing a family of period- 2 cycles with a constant and small distance $\left(\epsilon=10^{-4}\right.$ ) between the end points $u(1)$ and $v(1)$, namely by continuing a solution family of Eqs. (8a)-(8h) plus the following boundary condition

$$
\left(u_{1}(1)-v_{1}(1)\right)^{2}+\left(u_{2}(1)-v_{2}(1)\right)^{2}-\epsilon^{2}=0 .
$$

The corresponding bifurcation curve in the $\left(\lambda_{B}, \lambda_{T}\right)$ plane is shown in Fig. 5 and separates region 2 from region 3 , where the chaotic regime is obligate.

The bifurcation diagram of Fig. 5 can be qualitatively interpreted by saying that forest fires can be chaotic only if $\lambda_{B}$ and $\lambda_{T}$ are remarkably small. Since these two parameters identify the residual bush and tree biomasses after a fire, we know from the morphology of the various types of forests that they are both particularly small only in Mediterranean forests. Thus, the conclusion one can draw from the bifurcation diagram of Fig. 5 is that fire regimes of Mediterranean forests should be chaotic, while savannas and boreal forests should have periodic fires. This confirms the validity of the model as well as that of the bifurcation analysis. 


\section{Concluding remarks}

In this paper we have studied the behavior of a continuous-time model for forest fire prediction [12]. The model is a second-order impact model where the growth of bush and trees is a continuous and slow dynamic process, while the fire is a discontinuous event that occurs when the mix of bush and trees reach a specified manifold, called pre-fire manifold. For suitable values of the parameters the model predicts periodic fires like those occurring in savannas and boreal forests, while for other values it predicts chaotic fire regimes of the kind observed in most Mediterranean forests. Mathematically speaking, the transition from chaotic to periodic fire regimes is due to a border collision bifurcation [1-6], which can be easily detected and then continued by solving a standard boundary-value problem.

The result of our bifurcation analysis are interesting because they complement the simulation study carried out in [12]. The bifurcation diagrams reported in the paper point out only two bifurcations, namely a subcritical flip bifurcation and a border collision bifurcation. Since the model is an impact system, bifurcation of periodic orbits grazing the pre-fire manifold are also possible [20]. However, they have been intentionally excluded since our aim was to discuss only the simplest mechanism responsible of the transition from chaotic fire regimes of Mediterranean forest to cyclic regimes of savannas and boreal forests.

\section{References}

[1] M.I. Feigin, Doubling of the oscillation period with $C$-bifurcations in piecewise continuous systems, PMM J. Appl. Math. Mech. 34 (1970) 861-869.

[2] M.I. Feigin, On the generation of sets of subharmonic modes in a piecewise continuous system, PMM J. App. Math. Mech. 38 (1974) 810-818.

[3] M.I. Feigin, On the structure of $C$-bifurcation boundaries of piecewise continuous systems, PMM J. App. Maths. Mechs. 42 (1978) 820-829.

[4] H.E. Nusse, J.A. Yorke, Border-collision bifurcations including period two to period three for piecewise smooth systems, Physica D 57 (1992) 39-57.

[5] H.E. Nusse, J.A. Yorke, Border-collision bifurcations for piecewise smooth one-dimensional maps, Int. J. Bifurcat. Chaos 5 (1995) 189-207.

[6] H.E. Nusse, E. Ott, J.A. Yorke, Border-collision bifurcations: An explanation for observed bifurcation phenomena, Phys. Rev. E 49 (1994) 1073-1076.

[7] A.F. Filippov, Differential equations with discontinuous right-hand side. In American Mathematical Society Translations, Series 2, American Mathematical Society, 1964, pp. 199231.

[8] A.F. Filippov, Differential Equations with Discontinuous Righthand Sides, Kluwer Academic Publishers, Dordrecht, 1988.

[9] D. Liberzon, Switching Systems and Control, Birkhüser, 2003.

[10] F. Dercole, Border collision bifurcations in the evolution of mutualistic interactions, Int. J. Bifurcat. Chaos, in press. 
[11] B. Brogliato, Nonsmooth Mechanics, Springer Verlag, New York, 1999.

[12] S. Maggi, S. Rinaldi, A second-order impact model for forest fire regimes, Ecol. Lett., submitted for publication.

[13] W.C. Bessie, E.A. Johnson, The relative importance of fuels and weather on fire behavior on subalpine forests, Ecology 76 (1995) 747-762.

[14] R. Casagrandi, S. Rinaldi, A minimal model for forest fire regimes, Am. Nat. 153 (1999) $527-$ 539.

[15] S.H. Strogatz, Nonlinear Dynamics and Chaos, Addison-Wesely, 1994.

[16] Yu.A. Kuznetsov, Elements of Applied Bifurcation Theory, second ed., Springer Verlag, Berlin, 1998.

[17] E.J. Doedel, H.B. Keller, J.-P. Kernévez, Numerical analysis and control of bifurcation problems: (I) Bifurcation in finite dimensions, Int. J. Bifurcat. Chaos 1 (1991) 493-520.

[18] E.J. Doedel, H.B. Keller, J.-P. Kernévez, Numerical analysis and control of bifurcation problemsx problems: (I) Bifurcation in finite dimensions, Int. J. Bifurcat. Chaos 1 (1991) 745 772.

[19] E. Doedel, A. Champneys, T. Fairgrieve, Yu.A. Kuznetsov, B. Sandstede, X. Wang, AUTO97: continuation and bifurcation software for ordinary differential equations (with HOMCONT), Department of Computer Science, Concordia University, Montreal, QC, 1997.

[20] M. Bernardo di, M.I. Feigin, S.J. Hogan, M.E. Homer, Local analysis of $C$-bifurcations in ndimensional piecewise smooth dynamical systems, Chaos Soliton. Fract. 10 (1999) 1881-1908. 